\title{
The emergence of strange metal and topological liquid near quantum critical point in a solvable model
}

\author{
Eunseok Oh, Taewon Yuk and Sang-Jin Sin \\ Department of Physics, Hanyang University, \\ Seoul 04763, South Korea \\ E-mail: lspk.lpg@gmail.com, tae1yuk@gmail.com, sangjin.sin@gmail.com
}

\begin{abstract}
We discuss quantum phase transition by a solvable model in the dual gravity setup. By considering the effect of the scalar condensation on the fermion spectrum near the quantum critical point(QCP), we find that there is a topologically protected fermion zero mode associated with the metal to insulator transition. Unlike the topological insulator, our zero mode is for the bulk of the material, not the edge. We also show that the strange metal phase with T-linear resistivity emerges at high enough temperature as far as a horizon exists. The phase boundaries calculated according to the density of states allow us understanding the structures of the phase diagram near the QCP.
\end{abstract}

KEYWORDS: Holography and condensed matter physics (AdS/CMT), Spontaneous Symmetry Breaking, Topological States of Matter

ARXIV EPRINT: 2103.08166 


\section{Contents}

1 Introduction 1

2 The fermion zero mode in AdS 2

3 The topological insulator in AdS 4

3.1 Topology of JR solution on the half line 6

$\begin{array}{ll}3.2 & \text { Appearance of topological liquid }\end{array}$

4 Phase diagrams near the QCP $\quad 7$

4.1 The half width 8

4.2 Phase boundaries 9

4.3 A field theory model 11

5 Discussion 11

5.1 Chiral symmetry and its breaking in the AdS/CFT 11

5.2 When the back reaction can be neglected? 12

A Spectrum and the zero modes with scalar order for $g=-1 \quad 13$

A.1 Case 1: $\Phi=M z^{2} \quad 14$

A.2 Case 2: $\Phi=M_{0} z \quad 15$

B Analytic expression for $\Gamma(T) \quad 16$

$\begin{array}{lll}\text { B.1 Case } m=0 & 17\end{array}$

$\begin{array}{ll}\text { C Comment on the conformal factor } z^{ \pm m} & 18\end{array}$

$\begin{array}{ll}\text { D Localization of zero modes at the AdS boundary } & 19\end{array}$

E Existence of fermion zero mode for $m^{2} \neq-2 \quad 19$

\section{Introduction}

The quantum critical point (QCP) is a door to understanding strongly correlated systems [1], where the basic building blocks of the matter lose particle characters by the strong correlation so that usual field theory is difficult to apply. The gravity dual description [2-4] might work there due to the striking similarities between the QCP and the black hole: both get the universality by the information loss and both can be assigned with transport coefficients [5-7]. However, too much information is lost at the QCP to identify the physical system from the observed data so that informations off the QCP are essential 
for it. Such motivation led our recent study on the effect of the symmetry breaking on the fermion spectral function in [8]. We found features like Fermi arc of semi-metals, flat band [9] and nodal lines [10] as well as the gap and pseudo gap [11, 12]. What we found was rather surprising since we did not intend it and the topology is known to be associated with band structure rather than the correlation which makes the band structure fuzzy.

In this paper, we will show that when the fermion couples to the scalar with condensation in the holographic bulk space, the fermion can get a topologically protected zero mode localized at the AdS boundary, hence at the bulk of the material, and it can be used to provide an analytic solution to the prototype quantum transition like that of metal to insulator.

\section{The fermion zero mode in AdS}

Let the bulk fermion $\psi$ be the dual field to the boundary fermion $\chi$ and $\Phi^{I}$ be the dual bulk field of the operator $\bar{\chi} \Gamma^{I} \chi$, where $I$ is an index set for arbitrary type of tensor: $I=\left\{\mu_{1} \mu_{2} \cdots\right\}$ or $\left\{5 \mu_{1} \mu_{2} \cdots\right\}$ and the dot means the Lorentz contraction. We can encode the effect of the symmetry breaking on the spectrum of the fermions by considering the coupling $\bar{\psi} \Phi \cdot \Gamma \psi$ where $\Phi$ becomes a classical field under the symmetry breaking. Our model is given by the action given by the sum $S=S_{\Phi}+S_{\psi}+S_{\mathrm{bdy}}$, where

$$
\begin{aligned}
S_{\Phi} & =\int d^{d+1} \sqrt{-g}\left(D_{\mu} \Phi^{2}-m_{\Phi}^{2} \Phi^{2}\right), \\
S_{\psi} & =\int d^{d+1} \sqrt{-g} x i \bar{\psi}\left(\Gamma^{\mu} \mathcal{D}_{\mu}-(m+g \Phi)\right) \psi, \\
S_{\text {bdy }} & =i \int_{\partial M} d^{d} x \sqrt{-h} \bar{\psi} \psi .
\end{aligned}
$$

in the fixed metric and gauge field background $g_{\mu \nu}, A_{\mu}$. Our action is a local field theory in the bulk, and we do not care whether its dual theory is strictly local field theory at the boundary, because the strongly correlated boundary systems are not necessarily described by a local field theory in the boundary due to the quantum entanglement of such manybody system. However, we assume that $\Phi_{I} \bar{\psi} \Gamma^{I} \psi$, in leading order is dual to the boundary operator $\phi_{I}^{s} \bar{\chi} \Gamma^{I} \chi$, where $\phi_{I}^{s}$ is the source field of the operator $\bar{\chi} \Gamma^{I} \chi . \Phi_{I}$ is the 'extension' of $\phi_{I}^{s}$ according to the standard dictionary.

In this paper, we use the most canonical and simplest AdS black hole metric,

$$
d s^{2}=-\frac{f(z)}{z^{2}} d t^{2}+\frac{1}{z^{2} f(z)} d z^{2}+\frac{1}{z^{2}} \sum_{i=1}^{d-1} d x_{i}^{2}
$$

where $f(z)=1-\left(z / z_{H}\right)^{d}$ for $\operatorname{AdS}_{d+1}$ and $z_{H}$ is related to the temperature by $z_{H}=d / 4 \pi T$. We take $d=3$ throughout this paper unless mentioned as otherwise.

The fermion equation of motion is given by

$$
\left(\Gamma^{\mu} \mathcal{D}_{\mu}-m-g \Phi\right) \psi=0
$$


where $g= \pm 1$ and $\mathcal{D}_{\mu}=\partial_{\mu}+\frac{1}{4} \omega_{\mu a b} \Gamma^{a b}$. According to the choice of sign of $S_{\mathrm{bdy}}$, half of the bulk spinor degrees of freedom are projected out so that for \pm sign, only $\psi_{ \pm}$survive $[9,13]$. Following ref. [14], we use following Gamma matrices representation:

$$
\Gamma^{r}=\sigma^{3} \otimes \mathbf{1}_{2}, \quad \Gamma^{\mu}=\tau^{1} \otimes \gamma^{\mu}, \text { with } \gamma^{\mu}=\left\{i \sigma^{2}, \sigma^{1}, \sigma^{3}\right\} \text { for } \mu=0,1,2 .
$$

For $m_{\Phi}^{2}=-2$ the solution for the scalar in the zero temperature limit near the boundary is given by

$$
\Phi=M_{0} z+M_{1} z^{2}
$$

Due to the in-falling condition at the horizon, one of $M_{0}$ and $M_{1}$ is a $\mathrm{BC}$ and the other is determined as a consequence. If $M_{0}$ as source is zero, then $M_{1}$ is identified as the condensation corresponding to the spontaneous symmetry breaking. In general, $\Phi$ includes the non-zero source term $M_{0}$, which can correspond to the external driving force like doping parameter or pressure. In the zero temperature limit, the probe solution is good almost everywhere since the horizon is at the $z \rightarrow \infty$.

We can prove the existence of the zero modes by solving above Dirac equation explicitly to find the boundary Green functions whose poles give us the full information of the spectrum. The readers can find the derivation of the Green functions at zero temperature limit in the appendix A. Here we give just the result.

For $\Phi=M_{0} z$,

$$
\begin{aligned}
G_{R}^{g=1} & =\frac{(4 \mu)^{\frac{1}{2}+m} \Gamma(-2 m) \Gamma(1+m+\nu)}{\left(k^{2}-w^{2}\right) \Gamma(-m+\nu) \Gamma(1+2 m)} \gamma^{\mu} k_{\mu} \gamma^{t}, \\
G_{R}^{g=-1} & =\frac{(4 \mu)^{\frac{1}{2}+m} \Gamma(-2 m) \Gamma(1+m-\nu)}{\left(k^{2}-w^{2}\right) \Gamma(-m-\nu) \Gamma(1+2 m)} \gamma^{\mu} k_{\mu} \gamma^{t},
\end{aligned}
$$

where parameters $\mu, \nu$ are given by $\mu=k^{2}-w^{2}+M_{0}^{2}$ and $\nu=\frac{m}{\sqrt{1-\varepsilon^{\prime}}}$ with $\varepsilon^{\prime}=\frac{\omega^{2}-k^{2}}{M_{0}^{2}}$. The poles of the Green function are given by those of gamma function at the non positive integers. The massive spectrum is given by

$$
\omega^{2}-k^{2}=M_{0}^{2}\left(1-m^{2} /(n+m+1)^{2}\right), n=0,1,2 \cdots,
$$

for both $g= \pm 1$. From the Green functions, we can check that the zero mode pole of $G_{R}^{g=1}$ is cancelled, while that of $G_{R}^{g=-1}$ survives.

Similarly, for $\Phi=M_{1} z^{2}$, the Green functions are given by

$$
\begin{aligned}
G_{R}^{g=1} & =M_{1}^{-1 / 2+m} \frac{\Gamma\left(\frac{1}{2}-m\right) \Gamma\left(\frac{1}{2}+m-\varepsilon\right)}{2 \Gamma\left(\frac{1}{2}+m\right) \Gamma(1-\varepsilon)} \gamma^{\mu} k_{\mu} \gamma^{t}, \\
G_{R}^{g=-1} & =M_{1}^{-1 / 2+m} \frac{\Gamma\left(\frac{1}{2}-m\right) \Gamma(-\varepsilon)}{2 \Gamma\left(\frac{1}{2}+m\right) \Gamma\left(\frac{1}{2}-m-\varepsilon\right)} \gamma^{\mu} k_{\mu} \gamma^{t},
\end{aligned}
$$

where $\varepsilon=\omega^{2}-k^{2} /\left(4 M_{1}\right)$ and therefore the spectrum is given by

$$
\begin{array}{ll}
\omega^{2}-k^{2}=4 M_{1}(n+m+1 / 2) & \text { for } g=1, \\
\omega^{2}-k^{2}=4 M_{1} n, & \text { for } g=-1 .
\end{array}
$$

Here again the $n=0$ mode for $g=1$ is gapful but that for $g=-1$ is a gapless mode. 


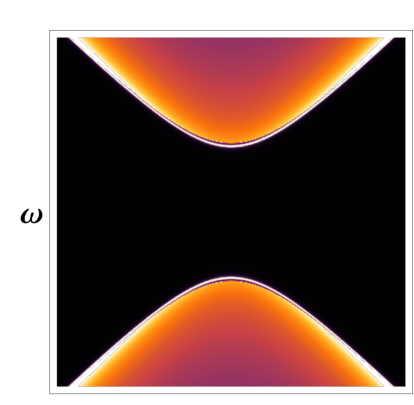

$\boldsymbol{k}$

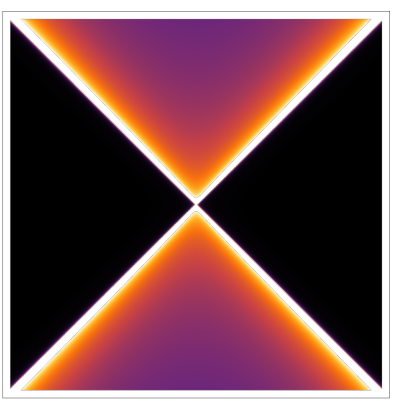

$\boldsymbol{k}$

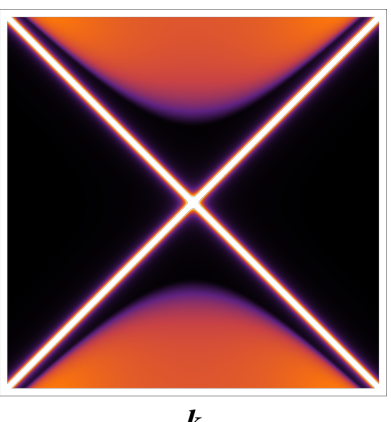

$\boldsymbol{k}$

(a) $g \Phi>0$, Gap

(b) $g \Phi=0, \mathrm{QCP}$

(c) $g \Phi<0$, Gapless

Figure 1. Spectral Functions for $\Phi=M_{0} z$. (a) for gapped phase, (b) for the QCP, (c) for the topological phase.

From these results, we see that for both $\Phi=M_{0} z$ and $\Phi=M_{1} z^{2}$, a gapless phase as well as the gaped phase emerges from the same QCP by invoking the scalar orders with different signs. See figure 1(a) and (c).

This is a surprising aspect of the holographic theory because such phenomena can never happen in flat spacetime or in weakly interacting system where the scalar order always introduces a gap. Such fermion zero mode has not been reported in holographic context as far as we know. Now, we are forced to ask, if an ordered state is gapless, how the order can be protected? We now want to understand its origin.

\section{The topological insulator in AdS}

The closest phenomena in the flat spacetime is the Jackiw-Rebbi (JR) fermion zero mode in the soliton background [15]. It is a solution of the Dirac equation $\left(\gamma^{\mu} \partial_{\mu}-\varphi\right) \psi=0$, where $\varphi$ changes sign across the domain wall. For such $\varphi$, the fermion has a normalizable zero mode, $\psi_{0}(x)=\exp \left(-\int d x \varphi\right)$, which is localized at the domain wall. Its stability is guaranteed by the boundary condition of $\varphi$, which makes it topological. See figure 2(a) top. There were flurries of activity last decade, under the name of the topological insulator after this solution is realized as the surface mode of condensed matter systems [16, 17]. In flat space, the soliton $\varphi$ can be realized by the sign changing fermion mass across the boundary of the material, $\varphi=m \cdot \operatorname{sign}(x)$, which in turn can be realized by the band inversion. See figure 2(a) bottom.

The gravity dual description has asymptotically Anti-de Sitter (AdS) space whose boundary is identified with the physical space in which materials are sitting. We will show that our zero mode solution is nothing but the AdS version of the Jackiw-Rebbi solution [15]. The argument can be greatly simplified in the approximation where gravity is $\mathrm{AdS}_{4}$ where the Dirac equation becomes

$$
\left[\Gamma^{z} \partial_{z}+i K_{\mu} \Gamma^{\mu}-\frac{m+g \Phi}{z}\right] \psi=0
$$




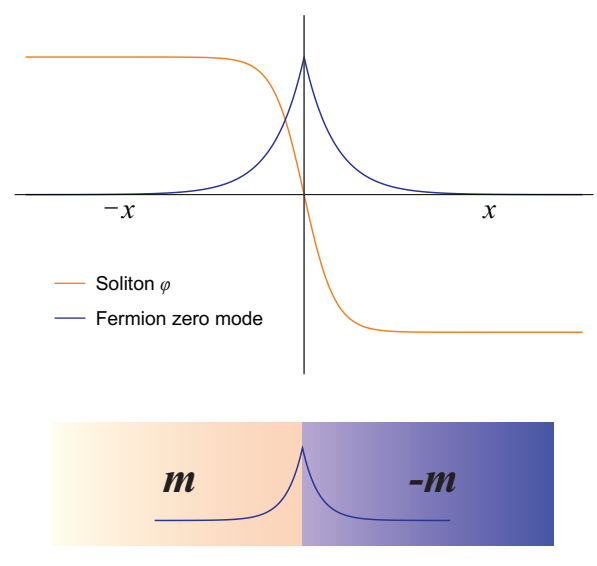

(a) Jackiw-Rebbi mode

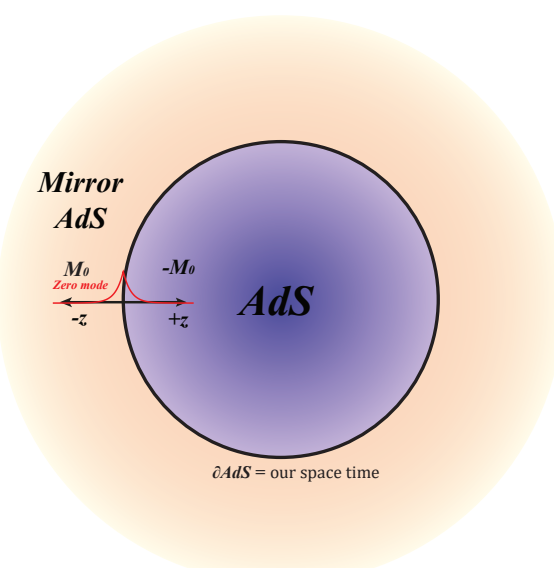

(b) JR mode in AdS

Figure 2. (a: top): Jackiw-Rebbi fermion zero mode in the soliton background. (a: bottom) Realization of the soliton $\varphi$ by sign changing mass. (b) $M_{0}$ corresponds to the $m$ in flat space. The boundary of AdS is the bulk of the physical space.

with $K^{\mu}=\left(\omega, k_{x}, k_{y}\right)$. Here the vielbeins in $\Gamma^{\mu}=e_{a}^{\mu} \Gamma^{a}$ were taken care of and all $\Gamma$ matrices in eq. (3.1) denote the constant ones $\Gamma^{a}$. Note that in the diagonal metric, the non-zero vielbein components are just square roots of the diagonal metric components. We construct the fermion zero mode in AdS by the separation of variables: $\psi_{0 \pm}(z, k)=\phi_{ \pm}(z) \chi_{0 \pm}(k)$, where $\phi_{ \pm}$is a scalar and the spinor $\chi_{0 \pm}(k)$ is defined by $\Gamma^{z} \chi_{0 \pm}(k)= \pm \chi_{0 \pm}(k)$. They satisfy

$$
\left[\mp \partial_{z}+\frac{m+g \Phi}{z}\right] \phi_{ \pm}=0, K_{\mu} \Gamma^{\mu} \chi_{0 \pm}(k)=0,
$$

respectively. The solution is given by

$$
\phi_{0 \pm}(z, x)=z^{ \pm m} \exp \left( \pm g \int_{0}^{z} d z^{\prime} \varphi\left(z^{\prime}\right)\right) \chi_{0 \pm}
$$

where $\varphi=\Phi / z$ for $z>0$. The standard (alternative) quantization means we project out $\psi_{0-}\left(\psi_{0+}\right)[9,18]$. We choose the standard quantization and negative $m$ because the spectral function $A \sim w^{2 m}$ and we want the spectral function to go zero when $\omega \rightarrow \infty$. Then for $g=-1$, the wave function are localized at $z=0$ as well as normalizable. Notice that $z^{ \pm m}$ factor does not make an issue of the normalizability because $|m|<1 / 2$ by the unitarity bound [18]. We see that the existence of the zero mode depends on whether the normalizable one survives the projection we choose.

At this point, we remind the reader that the essence of the Jackiw-Rebbi (JR) solution is the presence of the domain wall or a boundary where the mass changes its sign. The AdS has a natural boundary although we did not install it. To make the parallelism with JR solution more explicit, one may introduce the mirror AdS in the regime $z<0$. See figure 2(b). The sufficient condition for the normalizability of the zero mode in $-\infty<z<$ $\infty$, is $\varphi(-z)=-\varphi(z)$ which is clearly satisfied by

$$
\varphi=M_{0} \operatorname{sign}(z)+M_{1} z
$$


Each term can exist separately and their corresponding fermion zero modes are

$$
\psi_{0-}^{\left(M_{0}\right)}=|z|^{m} e^{-M_{0}|z|} \chi_{0-}, \quad \psi_{0-}^{(M)}=|z|^{m} e^{-\frac{1}{2} M_{1} z^{2}} \chi_{0-},
$$

respectively. Obviously these are localized at the boundary for $m<0$. The reader can read more explicit construction of the full solution in the appendix E. It should be reminded that the boundary of the AdS is the physical space where materials are sitting, therefore our zero mode describes a bulk phenomena, while the zero mode in a usual topological insulator (TI) describes a surface phenomena of the matter.

In the next subsection, we will discuss the topology of the solution and the possibility to see the topology without introducing the mirror AdS.

\subsection{Topology of JR solution on the half line}

One may ask if one can find the topological nature without extending the AdS to Mirror AdS. The question is natural since we did not use the Mirror AdS in the construction of the fermion zero mode in eq. (3.5). This is a subtle point that is worth while discussing in detail. However, we can ask the same question to the original JR solution. If we restrict the domain to half line $x \geq 0$, the wave function

$$
\psi \sim \exp \left[-\int_{0}^{x} \varphi\left(x^{\prime}\right) d x^{\prime}\right] \phi_{0}=e^{-m x} \phi_{0}
$$

with $\phi_{0}$ a constant spinor, is still a zero energy, normalizable solution of the Dirac equation which is localized at the boundary $x=0$, just like our case. So, where is the topology?

First, please be reminded that any domain wall solution can be viewed as the solution in $1+1$ where the topological current is

$$
j^{\mu}=\frac{1}{v} \epsilon^{\mu \nu} \partial_{\nu} \varphi
$$

where $v$ is a normalization constant defined as the difference of $\varphi$ values at the two boundary points.

The stability of fermion solutions comes from the topology of the soliton $\varphi$ whose winding number given by

$$
w=\frac{1}{v} \int_{S^{1}} \epsilon^{01} \partial_{x} \varphi(x)=\frac{1}{v}[\varphi(-\infty)-\varphi(+\infty)]=1 .
$$

If we restrict ourselves to the half line $x>0$, we get the same winding number as far as

1. We identify the half line as a circle by identifying two boundary points of the domain,

2. $\varphi(0) \neq \varphi(\infty)$ and we identify the end points to make the image of $\varphi$ as the circle.

A subtlety comes not from the restriction to $x>0$ but from the approximation of the soliton by the piecewise constant function $m(x)=\operatorname{sign}(x)$. Notice that the entire contribution of the winding number, when we use $m(x)=\operatorname{sign}(x)$, comes from $x=0$, because $\partial_{x} \operatorname{sign}(x)=$ $2 \delta(x)$. When the domain is the entire line as in the original JR case, we can intuitively see that the value of $m(x)$ at $x=0$ is 0 as the average value of $\varphi$ around $x=0$, so that the winding number is 1 .

We now look at the problem of topology of the JR solutions at the half line. 
(i) If we realize the kink by the $\varphi(x)=x$, there is no subtlety at all in getting $w=1$ in the half line. (Notice that the normalization factor $v$ depends on the ends points of $\varphi$.) This means that there is no subtlety in AdS for the condensation part $\Phi=M_{1} z^{2}$ without mirror AdS.

(ii) When we realize the kink by the piecewise constant mass function, the winding number is still 1 as far as we accept $m(0)=0$, because the discontinuity gives us the delta function. That is, the price to pay for the non-zero winding after restricting ourselves to the half line is just accepting $m(0)=0$. From the fermion side, the fermion configuration given in eq. (3.5) does NOT depends on the choice of $m(0)$ at all. Therefore stability of the fermion should not depends on it. The stability is checked by numerical experiment also.

In short, the topological nature of the JR soliton can be realized in the half line $x \geq 0$ as far as we install the discontinuity of the mass function at $x=0$. However, great deal of our intuition about the topology is lost in the half line case. So it is better to take the domain as the entire line by introducing the Mirror AdS.

\subsection{Appearance of topological liquid}

The gapless phase generated by the zero mode is dissipation free due to the topological protection, hence we call it as the topological liquid (TL). Notice that due to the presence of the quantization choice, our zero mode is a chiral in the sense of $\Gamma^{z}$ eigenvalue, which make sense independent of whether the boundary is $3+1$ or $2+1$ dimension. Such chiral and topological character of the zero mode makes the TL phase very different from the critical point. For example, one can see that, in figure 1(c), the zero mode is clearly separated from other modes, while figure 1(b) shows no separation of the massless spectrum from others. In mathematical term, the critical point has a brach cut type singularity for $m \neq 0$, while the topological phase has a pole type singularity.

\section{Phase diagrams near the QCP}

As the first application of the appearance of zero mode, we consider the phase diagram near the QCP. First, notice that we can associate a metal insulator transition at the QCP, because both the gap and the gapless features are coming out of a QCP by a single order parameter as we vary it passing the zero. Then, by adding the temperature, we can discuss the phase diagram near the quantum critical point. The finite temperature is described by the black hole geometry. The order parameter field $\Phi$ 's configuration for the pure AdS remains as a good approximation at finite temperature also, because the large $z$ region, where true solution deviates much from the probe solution, is cut out by the presence of the horizon.

The shape of density of states (DOS) is determined by the half width $\Gamma$, which depends on the order parameter and temperature. Therefore we can classify the phases according to $\Gamma$ as function of $T / M_{0}$. Since we have the analytic result for the latter, the entire phase 


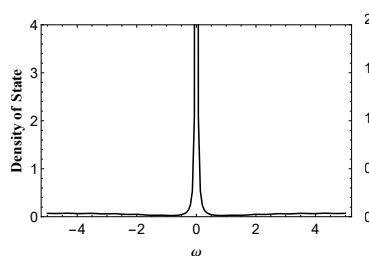

(a) Topological Liquid

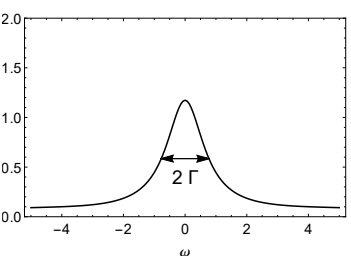

(b) Strange Metal

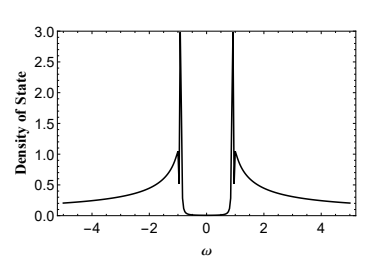

(c) Gap

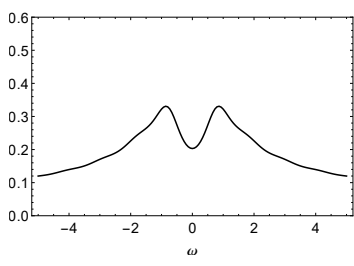

(d) Pseudo-gap

Figure 3. DOS for various phases $\Gamma(T)$ for $m=-0.3$ : (a,b) is along $g M_{0}=-2$ with $T=0.1,0.8$. (c,d) is along $g M_{0}=1$ with $T=0.01,0.15$ respectively.

diagram could be understood analytically. To visualize the typical density of states(DOS) of each phases, we calculated the DOS numerically. See the figure 3.

\subsection{The half width}

The half width $\Gamma(T)$ of fermion can be calculated within the probe approximation and the result is given by,

$$
\begin{aligned}
\Gamma(T) & =2 \pi T / \int_{0}^{1} \frac{d t F(t ; T)}{t(1-t)^{2 / 3}}, \\
F & =\sinh \left[\frac{4}{3}|m| \tan ^{-1} \sqrt{t}-\sum_{n=0}^{1} \frac{g M_{n}}{T^{1+n}} \beta_{n}(t)\right],
\end{aligned}
$$

with $\beta_{0}(t)=B\left(t ; \frac{1}{2}, \frac{1}{3}\right) / 2 \pi, \beta_{1}(t)=\left(3 / 8 \pi^{2}\right) B\left(t ; \frac{1}{2}, \frac{2}{3}\right)$. We take $-1 / 2<m<0$ for the bulk mass of the fermion. The point we want to make is that when the temperature is much larger than the order parameters, we have the linear- $T$ dependence,

$$
\Gamma \simeq \pi T / \gamma_{m}
$$

with $1 / \gamma_{m}=\frac{2 m}{3} B\left(\frac{1}{2}, \frac{1-2 m}{3}\right)_{3} F_{2}\left(\frac{1}{2}, \frac{3-4 m}{3}, \frac{3-2 m}{3} ; \frac{3}{2}, \frac{5-4 m}{6} ; 1\right)$. In the figure 4 , we plotted the half width given in eq. (4.1) as function of $T$ for $g M_{0}=-2$. The derivation and more general result can be found in the supplementary materials $\mathrm{C}$.

The emergence of strange metallicity $\Gamma(T) \sim T$ could be understood directly from the equation of the motion (3.1) by rescaling $z=\zeta / T$, without calculating $\Gamma(T)$ explicitly. The idea is that the Dirac equation contains the temperature dependence only through $w:=\omega / T$, if $\frac{M_{0}}{T} \ll 1$. If we call the spectral width in $w$ as $\Gamma_{0}$, it is independent of $T$. Then, $\Gamma(T)$ defined as the spectral width in $\omega$ is given by

$$
\Gamma(T)=\Delta \omega=\Gamma_{0} T,
$$

where $\Gamma_{0}=\Delta w$. This explains the appearance of the strange metal for temperature higher than the order parameter in figure $4(\mathrm{c}): \Gamma(T) \sim \hbar / \tau \sim T$ can be translated into the resistivity data $\rho \sim 1 / \tau \sim T$. The same argument can be applied to the equation describing the gauge field or metric field fluctuation in AdS space, and it gives the linear-T dependence for the width of the Drude peaks in the AC conductivity or in momentum dissipation rate. It works as far as there is a horizon in the background gravity. 


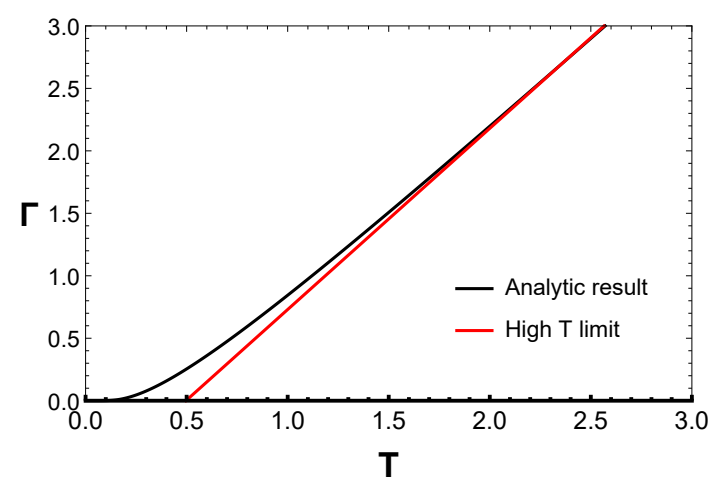

(a) $\Gamma(T)$ at $g M_{0}=-2$

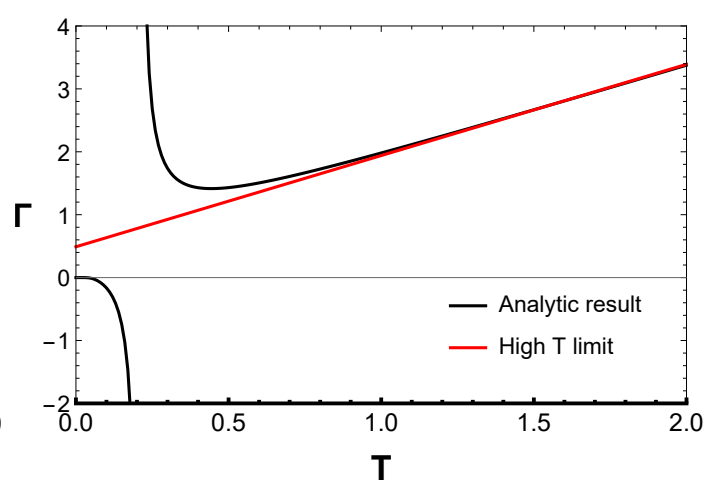

(b) $\Gamma(T)$ at $g M_{0}=+1$

Figure 4. $\Gamma$ as a function of $T$. For high temperature, $\Gamma(T) \sim T$ universally. (a) is for $g M_{0}>0$, (b) is for $g M_{0}<0$. In (b) $\Gamma<0$ at low $T$ means the appearance of the gap.

The strange metal has been discussed in the context of the holographic theory previously [19-24]. However, most of these literatures are based on the electric conductivity, and it has been argued only for the case where temperature is much smaller than any scale appearing in the theory [25], which is not what the data shows near QCP. This has been the difficulty in the holographic description of phase diagram near QCP. It is also worthwhile to notice that the argument based on the hydrodynamics has some difficulty also: while one should assume the slow momentum dissipation for the validity of hydrodynamics, which is just conservation law, the phenomena one want to explain is the ultra fast relaxation such that $\tau \sim 1 / T \ll T^{2} / E_{F}$.

\subsection{Phase boundaries}

The phase boundaries can be calculated by using eq. (4.1). We first introduce the key parameter $a$ by

$$
a\left(T, M_{0}\right):=\frac{\partial \log \Gamma}{\partial \log T}
$$

so that if $a\left(T, M_{0}\right)$ is constant along a curve $C$ in $T$ - $M_{0}$ plane, then $\Gamma \sim T^{a}$ on $C$. From eq. (4.1): if $\Phi=M_{0} z, \Gamma / T$ depends on the $T$ only through the combination $M_{0} / T$, therefore $a$ is constant on the straight line $T / M_{0}=c_{0}$. Completely parallel argument says that for $\Phi=M_{1} z^{2}$, the exponent $a$ is constant on any parabola $T / \sqrt{M_{1}}=c_{1}$. The phase diagram of the fermion theory with $\Phi=M_{0} z$ and that of $\Phi=M_{1} z^{2}$ are similar apart from the shape of the line of constant exponent. Since the phases near the QCP can be classified according to the power law $T^{a}$ of $\Gamma(T)$, the phase boundaries are along the lines of constant $a$. The resulting phase diagrams are figure $5(\mathrm{~b}, \mathrm{~d})$.

The structure of the phase diagram in $M_{0}-T$ plane can be explained as the competition of three phase regimes: i) Topological liquid(TL) around the negative $M_{0}$ axis where $a=\infty$, ii) the strange metallic phase around $T$ axis where $a=1$, iii)Gapped phase around the positive $M_{0}$ axis. 


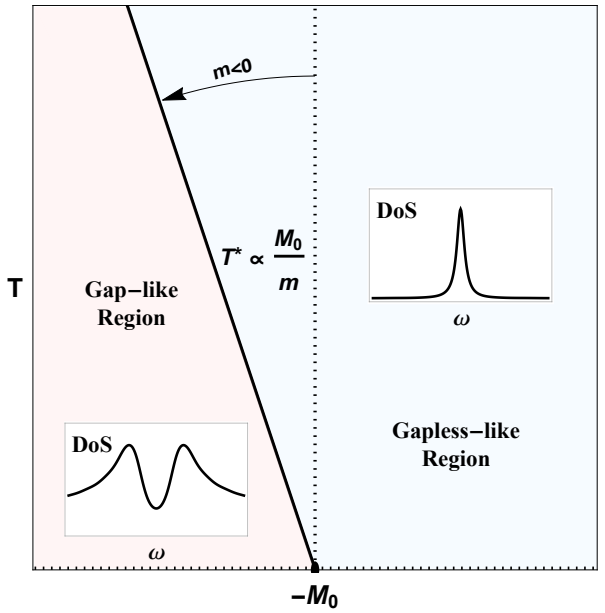

(a) structure of PD for $M_{0}$

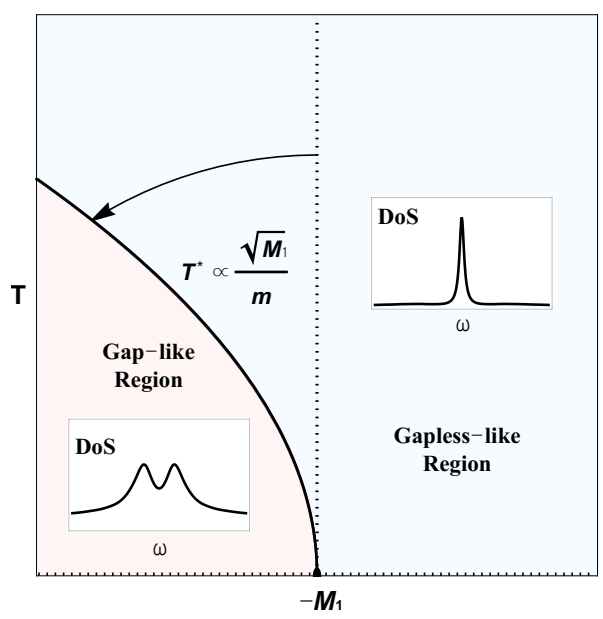

(c) structure of PD for $M_{1}$

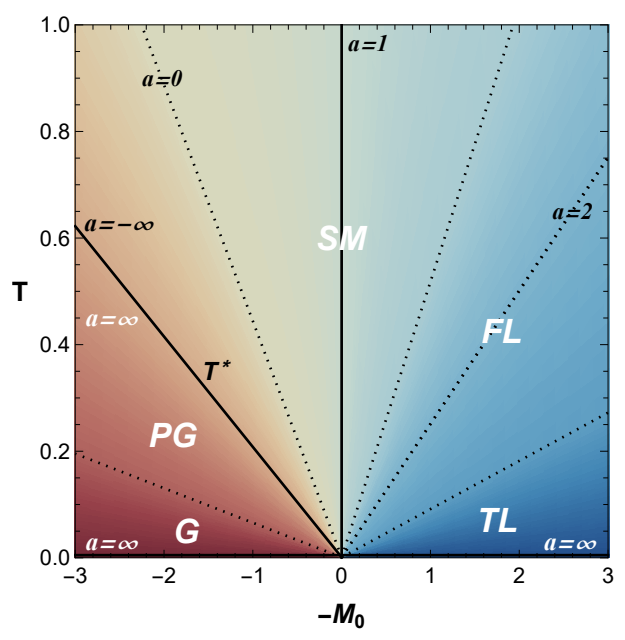

(b) phase diagram with $M_{0}$

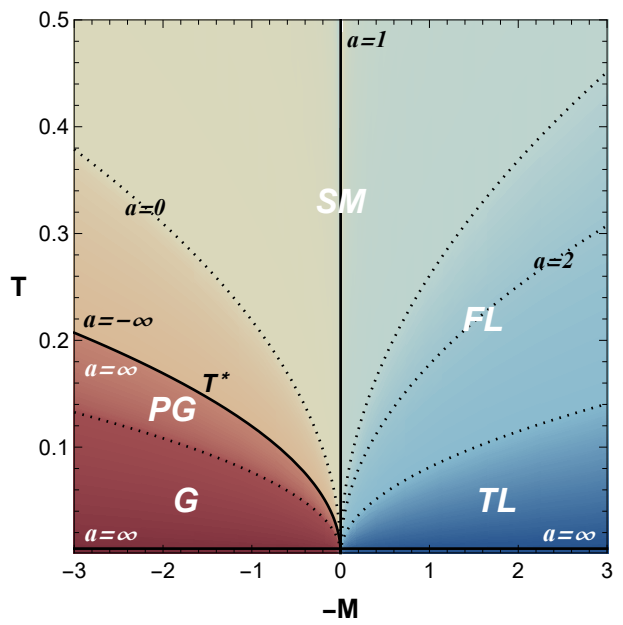

(d) phase diagram with $M_{1}$

Figure 5. True Phase transition exists only along $T_{*}$ lines. Others are crossover. In (b,d) 'Fermi liquid(FL) and strange metal(SM)' phases are around $a=2,1$ lines respectively.

The phase boundaries are mostly crossover except the $T^{*}$ line given by $T^{*}=M_{0} / \mathrm{m}$ with $-1 / 2<m<0$. It is the boundary between gapped and gapless regimes. When there is no order parameter, the strange metal appears even for the $T \rightarrow 0$ limit, which explains why the strange metallic phase is of fan shape starting from the QCP in the phase diagram. The figure 5(a) explains this idea. It would be interesting to compare figure 5(b,d) with the phase diagrams describing the semi-metals [26, 27] and heavy fermion systems [28] respectively, although we can not expect that they can be identified to specific materials, based on the universality near the QCP.

The presence of zero mode and its topological stability predicts the emergence of the topological liquid near zero temperature for a strongly correlated system with scalar source or condensation. On the other hand, from the holographic point of view, the strange metal 
is requested by the presence of horizon. Then between TL $(a=\infty)$ and SM $(a=1)$, there must be region of the Fermi liquid with $a=2$ by continuity.

\subsection{A field theory model}

Before we leave the phase diagram, it is worthwhile to discuss a related field theory model to see what we really achieved from the analysis above. Consider following field theory model [5],

$$
L=\phi \bar{\psi} \psi+r \phi^{2}+u \phi^{4}+(\partial \phi)^{2}+\bar{\psi} i \gamma^{\mu} \partial_{\mu} \psi
$$

For $r>0,\langle\phi\rangle=0$, and the fermion $\psi$ is massless, and free below the scale $\sqrt{r}$. For $r<0$, $\langle\phi\rangle \neq 0$ and the fermion is massive. The critical point is of Gross-Neveu type. There is an apparent similarity between the field theory model given in eq. (4.5) and our holographic one in the sense that both have the transition from gapful to gapless phases in $(r, T)$ phase diagram. However, in the phase diagram of weakly coupled theory, the strange metal (SM) phase (linear $\mathrm{T}$ in the resistivity or in the decay rate), can not appear, since no one got SM behavior by perturbative calculation. Nevertheless, the model (4.5) is truly intriguing, because in the presence of the Fermi Surface the above field theory model is a strongly coupled one in low energy [29-36], so that there is a possibility that in low energy it is in the same universality class with our holographic model. The reason why the linear in $\mathrm{T}$ behavior appeared in the decay rate $\Gamma(T)$ so naturally in our work is, of course, due to the gravity set up: the presence of the horizon and the structure of differential equation does everything as we have shown earlier.

\section{Discussion}

\subsection{Chiral symmetry and its breaking in the AdS/CFT}

It is interesting to notice that the scalar is associated with the chiral symmetry breaking in the bulk. But there is no chiral symmetry in $2+1$ boundary, since Lorentz group of $2+1$ does not contain two copies of $\mathrm{SU}(2)$. On the other hand, the symmetry group should be the same if the bulk and boundary theories are dual to each other. Therefore without a definition of chiral symmetry which works for both even and odd dimension, there would be a crash in the symmetry correspondence.

The way to solve this problem is to define the chiral symmetry in the odd dimension by introducing two flavors in the boundary and consider the chiral symmetry operation as the $\mathrm{U}(1)$ rotation with opposite charges for the two flavors. In our construction, we will use 2-component (Weyl) spinors for the boundary fermions for both AdS4 and AdS5.

First we discuss AdS5 which has chiral symmetry at the boundary. Each of 4-component Dirac fermions can be decomposed into two Weyl fermions $\chi_{1}, \chi_{2}$. Then, the chiral symmetry in 4 dimension is the rotation of them defined as

$$
\chi_{1} \rightarrow e^{i \alpha} \chi_{1}, \quad \chi_{2} \rightarrow e^{-i \alpha} \chi_{2} .
$$

Now, there are two 4-component fermions $\psi_{1}, \psi_{2}$ in AdS5 bulk. Out of four components of a bulk spinor $\psi$, two of them are projected out by the choice of 'quantization'. In the 
representation where $\Gamma^{\underline{z}}=\operatorname{diag}(1,1,-1,-1)$, the standard quantization keep the upper two components $\psi_{+}$, while the alternative quantization keeps the lower ones $\psi_{-}[9,18]$.

We now embed $\chi_{1}$ as the upper two components of $\psi_{1}$ and $\chi_{2}$ as the lower two components of $\psi_{2}$, namely,

$$
\psi_{1+}=\chi_{1}, \quad \psi_{2-}=\chi_{2} .
$$

After projections defined by the standard (S) quantization for $\psi_{1}$ and the alternative one (A) for $\psi_{2}$, degrees of freedom precisely match between the bulk and boundary. Furthermore, such correspondence induces a flavor rotation for the bulk fermions in AdS5:

$$
\psi_{1} \rightarrow e^{i \alpha} \psi_{1}, \quad \psi_{2} \rightarrow e^{-i \alpha} \psi_{2}
$$

So it is natural to call such symmetry operation as the 'chiral' rotation in the AdS5 bulk. In this way the 'chiral symmetry' in the bulk of AdS5 is induced by that of the boundary.

Now we consider $\mathrm{AdS}_{4}$. Here the boundary is $2+1$ dimension and there is no notion of chirality defined by the Lorentz group and parity. So introduce two fermions $\chi_{1}, \chi_{2}$, each of which has two components. Now embed the boundary fermions into that of the 4component bulk fermion exactly as the same as the AdS5 case, namely, $\psi_{1+}=\chi_{1}, \quad \psi_{2-}=$ $\chi_{2}$. Now, notice that in $\mathrm{AdS}_{4}$ bulk, there is a well defined chiral symmetry given by

$$
\psi_{ \pm} \rightarrow e^{ \pm i \alpha} \psi_{ \pm}
$$

which induces the flavor rotation in AdS4:

$$
\chi_{1} \rightarrow e^{i \alpha} \chi_{1}, \quad \chi_{2} \rightarrow e^{-i \alpha} \chi_{2}
$$

Such flavor rotation can be called as 'chiral rotation' in the $2+1$ boundary.

Notice that in the above discussion of the chiral symmetry for the AdS/CFT, essentially the same construction works both for $\mathrm{AdS}_{4}$ and $\mathrm{AdS}_{5}$. If we intended to show the conservation of such a symmetry, the problem would be much more non-trivial because a global symmetry should be gauged to be preserved in quantum gravity [37]. Here, however, we consider the broken symmetry, we can stop here. It is not surprising even in the case a global symmetry be spontaneously broken by the strong interaction.

What happen if there is only one flavor in $2+1$ boundary of $\mathrm{AdS}_{4}$ ? From AdS/CFT point of view, the theory is chiral in the sense that out of two chiral fermions of AdS4, only one has its boundary dual partner. Such a theory is chiral, that is, the 'chiral symmetry' is broken.

\subsection{When the back reaction can be neglected?}

We considered the probe limit of matter fields in this paper. Especially we should ask when the contribution from the fermions can be neglected in the equation of motion for the scalar. For high electron density, the back reaction is essential. Here, however, electrons couple weakly, so that Fermi liquid theory may be enough. This is because high density leads to the weak effective coupling. There is an approach called 'electron star' where one counts the electron contribution to the gravity. Indeed, many of its properties show those 
of the Fermi liquid theory. In fact, the strongly coupled nature of the electrons are coming when the density is low or the band width is small. Since we want to describe the strongly coupled electrons, we can describe it in the probe limit.

We conclude with following comment: the presence of the zero modes is related to the fact that the gravity dual use the asymptotically anti de Sitter space which must have a boundary, and the appearance of the strange metal is associated with the presence of the black hole horizon.

\section{A Spectrum and the zero modes with scalar order for $g=-1$}

Here we show the presence of the zero mode by working out the full spectrum. Our fermion action is given by the sum $S=S_{g, A, \Phi}+S_{\psi}+S_{b d r y}$, where

$$
\begin{aligned}
S_{g, A, \Phi} & =\int d^{d+1} x \sqrt{-g}\left(R+\frac{6}{L^{2}}-\frac{1}{4} F_{\mu \nu}^{2}+D_{\mu} \Phi_{I}^{2}-m_{\Phi}^{2} \Phi^{2}\right) \\
S_{\psi} & =\int d^{d+1} \sqrt{-g} x i \bar{\psi}\left(\frac{1}{2} \Gamma^{\mu}\left(\overrightarrow{\mathcal{D}}_{\mu}-\overleftarrow{\mathcal{D}}_{\mu}\right)-(m+g \Phi)\right) \psi \\
S_{\mathrm{bdy}} & = \pm \frac{i}{2} \int_{\partial M} d^{d} x \sqrt{-h} \bar{\psi} \psi .
\end{aligned}
$$

This action give the complete dynamics of all the fields including the Dirac field. According to the choice of sign of $S_{\mathrm{bdy}}$, half of the bulk spinor degrees of freedom are projected out so that for $+(-)$ sign, only $\psi_{+}\left(\psi_{-}\right)$survive and this choice of the boundary action is called standard (alternative) quantization $[9,13]$. In this paper we use the simplest AdS black hole metric,

$$
d s^{2}=-\frac{1}{L^{2}} \frac{f(z)}{z^{2}} d t^{2}+\frac{L^{2}}{z^{2} f(z)} d z^{2}+\frac{1}{L^{2} z^{2}} d x_{i}^{2}, \quad f(r)=1-\left(\frac{z}{z_{H}}\right)^{d}
$$

where the horizon radius is related to the temperature by $z_{H}=d / 4 \pi T$ and we set $L=1$. If we define $\phi_{ \pm}(z)$ by $\psi_{ \pm}=\left(-\operatorname{det} g g^{z z}\right)^{-1 / 4} e^{-i w t+i k_{i} x^{i}} \phi_{ \pm}(z)$, the $\phi$ satisfies

$$
\left[\partial_{z}+\frac{1}{\sqrt{f}}\left(i K_{\mu} \Gamma^{\mu}+\frac{m+g \Phi}{z}\right) \Gamma^{z}\right] \phi=0, \text { with } K_{\mu}=\left(-\omega / \sqrt{f}, k_{x}, k_{y}\right) .
$$

Following the standard dictionary of AdS/CFT for the $p$-form bulk field $\Phi$ dual to the operator $O$ with dimension $\Delta$, its mass is related to the operator dimension by

$$
m_{\Phi}^{2}=-(\Delta-p)(d-\Delta-p)
$$

and asymptotic form near the boundary is

$$
\Phi=\Phi_{0} z^{d-\Delta-p}+\left\langle O_{\Delta}\right\rangle z^{\Delta-p} .
$$

We use following Gamma matrices representation [14],

$$
\Gamma^{r}=\sigma^{3} \otimes \mathbf{1}_{2}, \quad \Gamma^{\mu}=\tau^{1} \otimes \gamma^{\mu}, \text { with } \gamma^{\mu}=\left\{i \sigma^{2}, \sigma^{1}, \sigma^{3}\right\}, \text { for } \mu=0,1,2 .
$$




\section{A.1 Case 1: $\Phi=M z^{2}$}

First we consider the case $\Phi=M z^{2}$. Then the Dirac equation is equivalent to

$$
-\phi_{ \pm}{ }^{\prime \prime}+\left(M^{2} z^{2}+\frac{m(m \pm 1)}{z^{2}}+2 g M\left(m \mp \frac{1}{2}\right)\right) \phi_{ \pm}=\mathcal{E} \phi_{ \pm},
$$

where $\mathcal{E}=w^{2}-\vec{k}^{2}$. The solution to the eq. (A.9) is given by

$$
\begin{aligned}
\phi_{+} & =2^{\frac{1}{2}\left(\frac{1}{2}-m\right)} z^{-m} e^{-M \frac{z^{2}}{2}}\left(\mathbf{C}_{1+} U_{u}^{\frac{1}{2}-m}\left(M z^{2}\right)+\mathbf{C}_{2+} L_{-u}^{-\frac{1}{2}-m}\left(M z^{2}\right)\right) \\
\phi_{-} & =2^{\frac{1}{2}\left(\frac{1}{2}+m\right)} z^{m} e^{-M \frac{z^{2}}{2}}\left(\mathbf{C}_{1-} U_{v}^{\frac{1}{2}+m}\left(M z^{2}\right)+\mathbf{C}_{2-} L_{-v}^{-\frac{1}{2}+m}\left(M z^{2}\right)\right) \\
\text { with } \varepsilon & =\frac{w^{2}-\vec{k}^{2}}{4 M}, u=\frac{1}{2}(g-1)\left(m-\frac{1}{2}\right)-\varepsilon, v=\frac{1}{2}(g+1)\left(m+\frac{1}{2}\right)-\varepsilon, \\
U_{u}^{k}(z) & =z^{-u}{ }_{2} F_{0}\left(u, 1+u-k ; ;-z^{-1}\right), L_{u}^{k}(z)=\frac{\Gamma(k+1+u)}{\Gamma(k+1) \Gamma(u+1)}{ }_{1} F_{1}(-u, k+1 ; z)
\end{aligned}
$$

where $\mathbf{C}_{i \pm}$ are two component constant spinors and $U_{u}^{k}$ and $L_{u}^{k}$ are associated Laguerre, whose asymptotic behavior determines the normalizability of $\psi$. Since the Laguerre function $L_{u}^{k}$ in general contains $e^{M z^{2}}$ we need to set $\mathbf{C}_{2 \pm}=0$. The $z \rightarrow \infty$ behaviors are

$$
\begin{aligned}
& \phi_{+} \sim 2^{\frac{1}{4}-\frac{m}{2}} e^{-\frac{M z^{2}}{2}} z^{-2 u-m} \mathbf{C}_{1+} M^{-u}, \\
& \phi_{-} \sim 2^{\frac{1}{4}+\frac{m}{2}} e^{-\frac{M z^{2}}{2}} z^{-2 v+m} \mathbf{C}_{1-} M^{-v} .
\end{aligned}
$$

Then, $z \rightarrow 0$ behaviors are given by

$$
\begin{aligned}
\phi_{+} & \sim 2^{\frac{1}{4}-\frac{m}{2}}\left(z^{-m} B_{1+}+z^{m+1} B_{2+}\right), & \phi_{-} & \sim 2^{\frac{1}{4}+\frac{m}{2}}\left(z^{1-m} B_{1-}+z^{m} B_{2-}\right) \\
\text { where } B_{1+} & =\mathbf{C}_{1+} \frac{\Gamma(m+1 / 2)}{\Gamma(u+m+1 / 2)}, & B_{2+} & =\mathbf{C}_{1+} M^{1 / 2-m} \frac{\Gamma(-1 / 2-m)}{\Gamma(u)}, \\
B_{1-} & =\mathbf{C}_{1-} M^{1 / 2-m} \frac{\Gamma(m-1 / 2)}{\Gamma(u+m+g / 2)}, & B_{2-} & =\mathbf{C}_{1-} \frac{\Gamma(1 / 2-m)}{\Gamma(u+(g+1) / 2)} .
\end{aligned}
$$

The relation

$$
B_{2-}=i(2 m+1) \frac{\gamma^{\mu} k_{\mu}}{w^{2}-k^{2}} B_{2+}, \quad B_{1-}=i(2 m-1) \frac{\gamma^{\mu} k_{\mu}}{w^{2}-k^{2}} B_{1+}
$$

which was established in [14] still hold here in the presence of the interaction term. Then the Green Function $G_{R}$ is defined by

$$
G_{R}=-i S \gamma^{0}, \quad \text { with } \mathrm{S} \text { defined by } B_{2-}=S B_{1+} .
$$

Now we can write down Green functions for each sign of $g$.For $\Phi=M z^{2}$,

$$
\begin{aligned}
G_{R}^{g=1} & =M^{-1 / 2+m} \frac{\Gamma\left(\frac{1}{2}-m\right) \Gamma\left(\frac{1}{2}+m-\varepsilon\right)}{2 \Gamma\left(\frac{1}{2}+m\right) \Gamma(1-\varepsilon)} \gamma^{\mu} k_{\mu} \gamma^{t}, \\
G_{R}^{g=-1} & =M^{-1 / 2+m} \frac{\Gamma\left(\frac{1}{2}-m\right) \Gamma(-\varepsilon)}{2 \Gamma\left(\frac{1}{2}+m\right) \Gamma\left(\frac{1}{2}-m-\varepsilon\right)} \gamma^{\mu} k_{\mu} \gamma^{t} .
\end{aligned}
$$


The poles of the Green function are given by those of gamma function at the non positive integers so that the spectra are given by

$$
\begin{aligned}
& \omega^{2}-k^{2}=4 M(n+m+1 / 2), \text { for } g=1, \\
& \omega^{2}-k^{2}=4 M n, \text { for } g=-1,
\end{aligned}
$$

with $n=0,1,2, \cdots$. The first spectrum is gapful for any $n$, but the second one has the zero mode at $n=0$.

\section{A.2 Case 2: $\Phi=M_{0} z$}

Now we turn to the case $\Phi=M_{0} z$. The equation of motion for $\phi$ with scalar source $M_{0}$ is equivalent to

$$
-\phi_{ \pm}^{\prime \prime}+\left(\frac{m(m \pm 1)}{z^{2}}+M_{0}^{2}+g \frac{2 m M_{0}}{z}\right) \phi_{ \pm}=\mathcal{E} \phi_{ \pm}
$$

After fixing the coefficients to remove the divergent pieces in $z \rightarrow \infty$ limit, the solution is

$$
\begin{aligned}
\phi_{ \pm} & =e^{-\sqrt{\mu} z}(2 \sqrt{\mu} z)^{\mp m} U_{\mp m+g \nu}^{\mp 2 m}(2 \sqrt{\mu} z) \\
\mu & =k^{2}-w^{2}+M_{0}^{2}, \quad \nu=\frac{m}{\sqrt{1-\varepsilon^{\prime}}}, \quad \varepsilon^{\prime}=\frac{\omega^{2}-k^{2}}{M_{0}^{2}} .
\end{aligned}
$$

The $z \rightarrow 0$ behavior of the solution is

$$
\begin{aligned}
& \phi_{+} \sim z^{-m} \frac{(2 \sqrt{\mu})^{-m} \Gamma(1+2 m)}{\Gamma(1+m+g \nu)}+z^{1+m} \frac{(2 \sqrt{\mu})^{1+m} \Gamma(-1-2 m)}{\Gamma(-m+g \nu)} \\
& \phi_{-} \sim z^{1-m} \frac{(2 \sqrt{\mu})^{1-m} \Gamma(2 m-1)}{\Gamma(m+g \nu)}+z^{m} \frac{(2 \sqrt{\mu})^{m} \Gamma(1-2 m)}{\Gamma(1-m+g \nu)} .
\end{aligned}
$$

These data give the Green functions for $\Phi=M_{0} z$ :

$$
\begin{aligned}
G_{R}^{g=1} & =\frac{(4 \mu)^{\frac{1}{2}+m} \Gamma(-2 m) \Gamma(1+m+\nu)}{\left(k^{2}-w^{2}\right) \Gamma(-m+\nu) \Gamma(1+2 m)} \gamma^{\mu} k_{\mu} \gamma^{t}, \\
G_{R}^{g=-1} & =\frac{(4 \mu)^{\frac{1}{2}+m} \Gamma(-2 m) \Gamma(1+m-\nu)}{\left(k^{2}-w^{2}\right) \Gamma(-m-\nu) \Gamma(1+2 m)} \gamma^{\mu} k_{\mu} \gamma^{t}
\end{aligned}
$$

where parameters $\mu, \nu$ are given by $\mu=k^{2}-w^{2}+M_{0}^{2}$ and $\nu=\frac{m}{\sqrt{1-\varepsilon^{\prime}}}$ with $\varepsilon^{\prime}=\frac{\omega^{2}-k^{2}}{M_{0}^{2}}$. Although these two look similar, there is a striking difference: notice that $\left(k^{2}-w^{2}\right) \Gamma(-m+$ $\nu) \approx-2 M_{0}^{2} / m$ near the lightcone $k^{2}=\omega^{2}$, therefore the apparent zero mode pole of $G_{R}^{g=1}$ is cancelled, while the zero mode of $G_{R}^{g=-1}$ survives. The massive particle spectra exist only for $m<0$ if $g=1$, while they exist only for $m>0$ if $g=-1$. In both cases, the massive tower is given by

$$
\omega^{2}-k^{2}=M_{0}^{2}\left(1-\frac{m^{2}}{(n+m+1)^{2}}\right), \quad n=0,1,2 \cdots .
$$

The spectra given in eq. (A.22) and (A.30) are the Kaluza Klein tower associated with the box character of AdS space, which gives an effective compactification. 


\section{B Analytic expression for $\Gamma(T)$}

To calculate the $\Gamma$ as a function of the temperature, we define the spectral function $\mathcal{A}$ by

$$
\mathcal{A}=\operatorname{Tr}\left(\operatorname{Im} G_{R}\right)
$$

and expand $\mathcal{A}$ for small $w$ by,

$$
\begin{aligned}
\mathcal{A} & =\mathcal{A}^{(0)}(1-\tau w+\cdots) \\
& =\mathcal{A}^{(0)}+w \mathcal{A}^{(1)}+\cdots
\end{aligned}
$$

Then the relaxation time $\tau$ is defined by the small $w$ expansion

$$
\tau=\frac{1}{\Gamma}=-\frac{\mathcal{A}^{(1)}}{\mathcal{A}^{(0)}}
$$

The Dirac equation (A.5) is equivalent to the flow equation for $\xi_{ \pm}$as follows

$$
\left(\partial_{z}+2 \frac{g \Phi+m / z}{\sqrt{f(z)}}\right) \xi_{ \pm}(z)=\left(\frac{w}{f(z)} \pm \frac{k}{\sqrt{f(z)}}\right) \xi_{ \pm}(z)^{2}+\left(\frac{w}{f(z)} \mp \frac{k}{\sqrt{f(z)}}\right)
$$

where $\xi_{+}=\frac{i y_{-}}{z_{+}}, \xi_{-}=-\frac{i z_{-}}{y_{+}}$and $\phi_{ \pm}=\left(y_{ \pm}, z_{ \pm}\right)^{T}$. The retarded Green function $G_{R}=$ $\left.z^{2 m} \operatorname{diag}\left(\xi_{+}, \xi_{-}\right)\right|_{z \rightarrow 0}$ [14]. From now on we set $k=0$, so that the equations for $\xi_{+}$and $\xi_{-}$ are the same for $k=0$ and we delete the lower index \pm from $\xi_{ \pm}$. Now, we expand the $\xi$ in $w$ up to first order,

$$
\xi(z)=\xi^{(0)}(z)+w \xi^{(1)}(z)+\cdots .
$$

Substituting the eq. (B.6) into eq. (B.5), the equations for the zero-th and first order in w expansion becomes

$$
\begin{aligned}
& \left(\partial_{z}+2 \frac{g \Phi+m / z}{\sqrt{f(z)}}\right) \xi^{(0)}(z)=0 \\
& \left(\partial_{z}+2 \frac{g \Phi+m / z}{\sqrt{f(z)}}\right) \xi^{(1)}(z)=\frac{1+\xi^{(0)}(z)^{2}}{f(z)} .
\end{aligned}
$$

The in-falling boundary conditions at the horizon implies $\xi^{(0)}\left(z_{H}\right)=i, \xi^{(1)}\left(z_{H}\right)=0$. We now can calculate the decay rate $\Gamma$ using

$$
\Gamma=-\frac{\mathcal{A}^{(0)}}{\mathcal{A}^{(1)}}=-\left.\frac{\operatorname{Im} \xi^{(0)}(z)}{\operatorname{Im} \xi^{(1)}(z)}\right|_{z \rightarrow 0} .
$$

Case $\boldsymbol{m} \neq \mathbf{0}$. A decay rate $\Gamma$ for $\operatorname{AdS}_{d+1}$ with $\Phi=M_{0} z+M_{1} z^{2}$ is given by,

$$
\begin{aligned}
\Gamma(T) & =\frac{2 \pi T}{\int_{0}^{1} d t(1-t)^{(1 / d-1)} F(t) / t}, \\
\text { with } F(t) & =-\sinh \left[\frac{4 m}{d} \tan ^{-1} \sqrt{t}+\frac{g M_{0} \beta_{0}(t)}{T}+\frac{g M_{1} \beta_{1}(t)}{T^{2}}\right],
\end{aligned}
$$




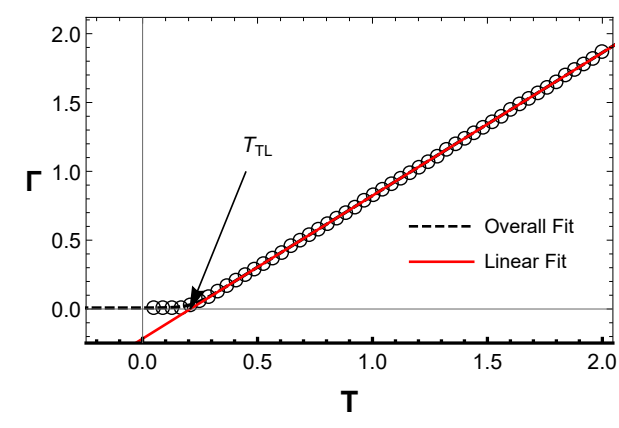

(a) $M=-2$

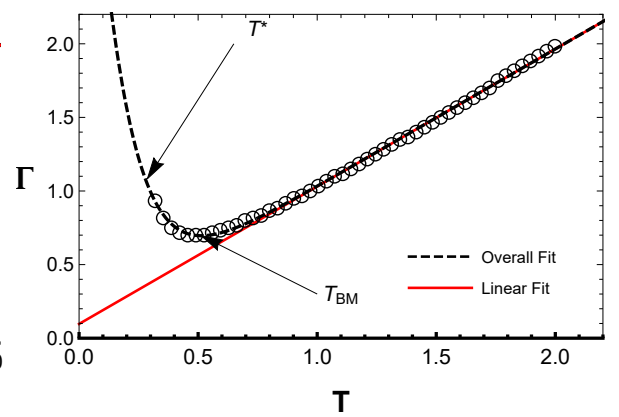

(b) $M=1$

Figure 6. $\Gamma(T)$ for $m=-0.4$ : for (a) $M_{1}=-2$ (b) $M_{1}=1$ respectively. Numerical $\Gamma$ is defined by a half width of spectral function at $w=0$.

where $\beta_{0}(t)=\frac{1}{2 \pi} B\left(t ; \frac{1}{2}, \frac{1}{d}\right), \quad \beta_{1}(t)=\frac{d}{8 \pi^{2}} B\left(t ; \frac{1}{2}, \frac{2}{d}\right)$. Notice that in this paper we take $-1 / 2<m<0$. This result explains the appearance of the strange metallicity at the critical point and near by region. At the criticality where the order parameters are zero, the appearance of the strange metalicity is equivalent to the presence of the black hole horizon, which in turn is equivalent to the presence of the scrambling power of the chaotic fluctuation of the quantum critical point. However, the appearance of other phases is consequence of the symmetry breaking. Such competition of the strange metallicity, or chaos, and the order determines the shape of the phase diagram near the QCP. Notice that

$$
\Gamma \simeq \pi T / \gamma_{m, d}, \quad \text { at } T \gg \sqrt{M_{1}}, M_{0},
$$

with

$$
\gamma_{m, d}^{-1}=\frac{2 m}{d} B\left(\frac{1}{2}, \frac{1-2 m}{d}\right){ }_{3} F_{2}\left(\frac{1}{2}, \frac{1}{2}-\frac{2 m}{d}, 1-\frac{2 m}{d} ; \frac{3}{2}, \frac{1}{2}+\frac{1-2 m}{d} ; 1\right) .
$$

For $d=1,2$, we have simple result $\gamma_{d}=\frac{\pi}{2} \tan (m \pi)$. We used eq. (B.11) to calculate the phase boundaries.

One may want to compare above analytic result with numerical calculation to check its validity. The result is figure 6 , showing that our formula agrees with numerical calculation precisely.

\section{B.1 Case $m=0$}

For $m=0$, the spectral function $\mathcal{A}$ has non zero asymptotic value, i.e. $\lim _{\omega \rightarrow \infty} \operatorname{Im} G(\omega)=1$. Therefore, the usual definition does not work. To overcome, we define the Drude function $\mathcal{A}_{D}$ by $\mathcal{A}_{D}=\mathcal{A}-1$. Then the relaxation time $\tau$ is defined by the small $\omega$ expansion $\mathcal{A}_{D}(w) \sim \mathcal{A}_{D}(0)(1-\tau w+\cdots)$, so that $\tau=\left.\frac{1}{\left|\mathcal{A}_{\mathcal{D}}\right|} \frac{\partial \mathcal{A}_{\mathcal{D}}}{\partial w}\right|_{w=0}$. Obviously negative- $\Gamma$ can be interpreted as a measure of the gap. With this preparation, we get the following formula for $m=0$ for $A d S_{d+1}, d>1$ :

$$
\Gamma=2 \pi T \frac{\left(e^{\frac{\alpha g M_{0}}{T}}-1\right)}{\int_{0}^{1}(1-t)^{(1 / d-1)} \frac{\sinh \left(g M_{0} \beta_{0}(t) / T\right)}{t} d t},
$$




\begin{tabular}{|c|c|c|c|c|}
\hline $\mathrm{d}$ & 1 & 2 & 3 & 4 \\
\hline \hline$\gamma_{d}$ & 1 & 1.1662 & $\sqrt{3} \pi / 4$ & $\pi / 2$ \\
\hline$\gamma_{d}^{\prime}$ & $\frac{7}{5}$ & $\pi / 2$ & 1.7538 & 1.9468 \\
\hline
\end{tabular}

Table 1. $\gamma_{d}$ and $\gamma_{d}^{\prime}$ for $\operatorname{AdS}_{d+1}, \quad d=1,2,3,4$.

where $\alpha=\frac{1}{2 \pi} B\left(\frac{1}{2}, \frac{1}{d}\right), \quad \beta_{0}(t)=\frac{1}{2 \pi} B\left(t ; \frac{1}{2}, \frac{1}{d}\right)$. Notice that for the large $\mathrm{T}$, the width is also reduced to linear in $T: \Gamma \simeq \frac{\pi T}{\gamma_{d}}$, with $\gamma_{d}={ }_{3} F_{2}\left(\frac{1}{2}, \frac{1}{2}, 1-\frac{1}{d} ; \frac{3}{2}, \frac{1}{2}+\frac{1}{d} ; 1\right)$.

Similarly, for the condensation, the decay rate with the scalar condensation is

$$
\Gamma=2 \pi T \frac{\left(e^{\frac{\alpha^{\prime} g M_{1}}{T^{2}}}-1\right)}{\int_{0}^{1}(1-t)^{(1 / d-1)} \frac{\sinh \left(g M_{1} \beta^{\prime}(t) / T^{2}\right)}{t} d t}
$$

where $\alpha^{\prime}=\frac{d}{8 \pi^{2}} B\left(\frac{1}{2}, \frac{2}{d}\right), \beta^{\prime}(t)=\frac{d}{8 \pi^{2}} B\left(t ; \frac{1}{2}, \frac{2}{d}\right), \Gamma \simeq \frac{\pi T}{\gamma_{d}^{\prime}}$ in the large T limit, with

$$
\gamma_{d}^{\prime}=\sqrt{\pi} 2^{1-\frac{2}{d}} \frac{\Gamma\left(\frac{1}{2}+\frac{2}{d}\right)}{\Gamma\left(\frac{1}{2}+\frac{1}{d}\right)^{2}} \cdot{ }_{3} F_{2}\left(\frac{1}{2}, \frac{1}{2}, 1-\frac{2}{d} ; \frac{3}{2}, \frac{1}{2}+\frac{2}{d} ; 1\right) .
$$

We tabulated $\gamma_{d}$ and $\gamma_{d}^{\prime}$ in table 1 explicitly.

\section{Comment on the conformal factor $z^{ \pm m}$}

The point we want to make below is that $z^{ \pm m}$ is not the factor that counts probability of location but the conformal factor to embed the boundary theory into the AdS bulk, which we should delete in probability interpretation of locality. To see why this is so, we remind the basic dictionary of the AdS/CFT for scalar case: if a scalar operator of dimension $\Delta$ couple to the source $\phi_{0}(x)$, then the bulk field dual to the operator, $\Phi(z, x)$, is NOT given by the direct extension of the $\phi_{0}$. Due to the conformal structure of AdS space [3], we need to dress the source and response by the conformal factor $z^{\Delta_{\mp}}$ such that two independent solutions of the bulk field $\Phi$ are given by $\Phi(z, x)=\phi_{0}(x) z^{\Delta_{-}}(1+\cdots)$ and $\Phi(z, x)=\langle O\rangle z^{\Delta_{+}}(1+\cdots)$, where $\Delta_{ \pm}$are the solutions of $\Delta(\Delta-d)=m_{\Phi}^{2}$. The equation of motion for $\Phi$ is of second order so that the general solution is given by the linear combination of the two. Conversely, in other to read off the 'extended configuration of $\phi_{0}$ ' from the solution of the bulk equation of motion, we need to strip off the factor $z^{\Delta_{ \pm}}$from the first and second solutions. Therefore, we define the undressed physical source/response bulk field $\Phi_{u, s}, \Phi_{u, r}$ by dividing out the conformal factor from the corresponding solutions of the bulk equation of motion.

Similarly for the spinor fields $\psi=\left(\psi_{+}, \psi_{-}\right)^{T}$, we can define the undressed source and response functions by $\psi_{ \pm}=z^{\Delta_{\mp}} \psi_{u \pm}$ with $\Delta_{ \pm}=3 / 2 \pm m$. It is worthwhile to mention that the boundary Green functions are given by the ratio of these undressed wave functions for both bosons and fermions. Then we can show that the normalizable undressed wave function is localized at the boundary and only the zero mode has such property. 


\section{Localization of zero modes at the AdS boundary}

Coming back to the AdS plus mirror space, the undressed wave function for the zero mode is

$$
\psi_{0 u+} \simeq e^{-M_{0}|z|}, \text { for }-\infty<z<\infty .
$$

This is precisely the Jackiw-Rebbi's normalizable soliton solution localized at the domain wall $z=0$ where the kink configuration is realized by term $M_{0} \operatorname{sign}(z)$. It also means that our zero mode can be considered as the edge state of a virtual topological insulator. However, we should not forget that our zero mode describe the bulk mode since it is free to move along the boundary of the AdS, which is the bulk of the physical world. In all these discussion, we introduced the mirror AdS to take direct similarity of JR solution. However one should notice that all that is used is to have the refection symmetry of the wave equation and a non-vanishing Dirichlet boundary condition of the undressed wave function $\psi_{u \pm}$ at $z=0$.

For $\Phi=M_{1} z^{2}$ and the equation of motion (A.9) is invariant under $z \rightarrow-z$ without sign change of $M$. The ground state given by

$$
\psi_{+} \simeq e^{-M_{1} z^{2} / 2}
$$

which is the zero mode localized at the domain wall at $z=0$.

One might think that any state are localized the boundary of the AdS. Below, we will show only the ground state for $g=-1$ is localized while all other states are not. To see this, notice that

$$
\begin{gathered}
\psi_{u+}^{g=1}(z=0) \sim 2^{\frac{1}{4}-\frac{m}{2}} \frac{\Gamma\left(\frac{1}{2}+m\right)}{\Gamma(-n)}=0, \text { for any integer } n \geq 0, \\
\psi_{u+}^{g=-1}(z=0) \sim 2^{\frac{1}{4}-\frac{m}{2}} \frac{\Gamma\left(\frac{1}{2}+m\right)}{\Gamma(1-n)}>0, \text { for } n=0,
\end{gathered}
$$

which shows clearly the uniqueness of the zero mode for $g=-1$. For a massive mode with $n \geq 1$, the wave functions are not localized for two reasons: first, the wave functions vanish at $z=0$ and secondly they oscillate and penetrates into larger $z$ region. The larger is $n$, the deeper it penetrates. See the figure 7. As far as $M_{1}$ or $M_{0}$, which is the gap between the ground state and the others, is non-zero, there exists a fermion zero mode for $g=-1$ so we may say that the fermion zero mode is protected by the presence of the gap, therefore we call the symmetry broken phase with $g=-1$ as 'topological liquid'. The point we want to make is that the zero mode has a topological character, and as such, it has to do with a phenomena which looks like Fermi liquid but with an unusual stability.

\section{E Existence of fermion zero mode for $m^{2} \neq-2$}

The existence of fermion zero mode is shown for $m^{2}=-2$. Although the analytical work is possible to this value, one may ask whether these phenomena survive away from $m^{2}=-2$ ? We numerical study showed the presence of a fermion solution with sharp dual boundary spectrum, as you can see in the figure 8 . 


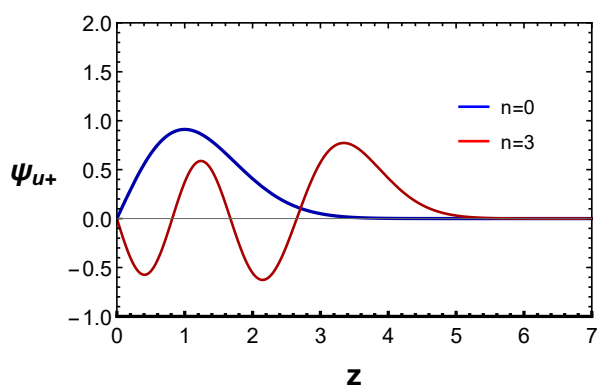

(a) $g=1$

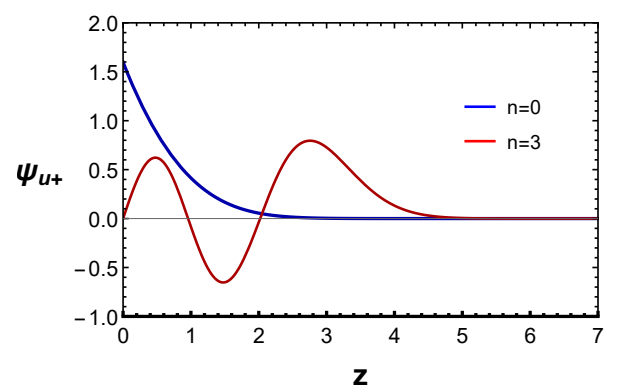

(b) $g=-1$

Figure 7. wave function for $M=1, m=0$ for various modes. Only the ground state for $g=-1$ state is localized at the boundary of the AdS.

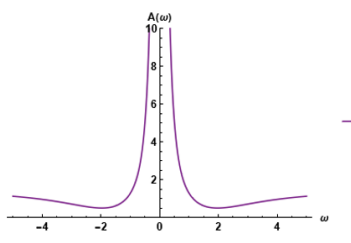

(a) $\Delta=0.5$

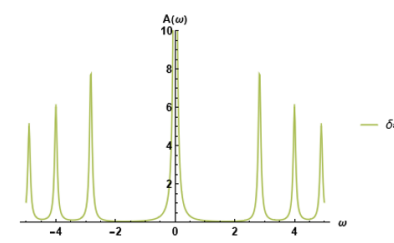

(d) $\Delta=2$

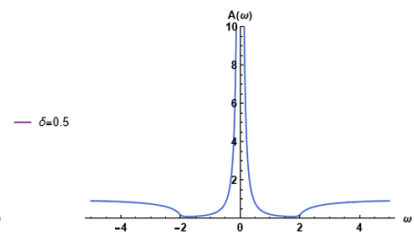

(b) $\Delta=1$

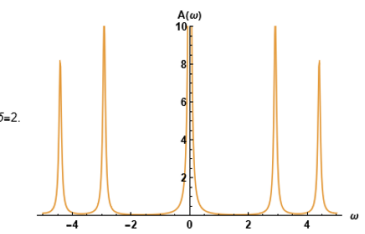

(e) $\Delta=2.5$

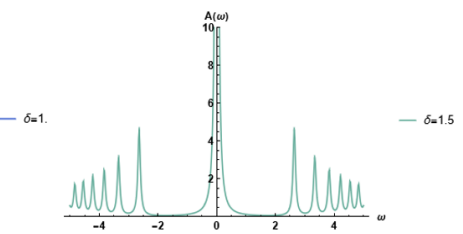

(c) $\Delta=1.5$

Figure 8. Spectral Functions for various $\Delta$ 's with $m^{2}=\Delta(\Delta-3)$. In all cases we can see the poles in the Green function, meaning the presence of the zero mode for all available values of $\mathrm{m}^{2}$.

\section{Acknowledgments}

This work is supported by Mid-career Researcher Program through the National Research Foundation of Korea grant No. NRF-2021R1A2B5B02002603. We thank the APCTP for the hospitality during the focus program, where part of this work was discussed.

Open Access. This article is distributed under the terms of the Creative Commons Attribution License (CC-BY 4.0), which permits any use, distribution and reproduction in any medium, provided the original author(s) and source are credited.

\section{References}

[1] S. Sachdev, Quantum Phase Transitions, second edition, Cambridge University Press, Cambridge U.K. (2011).

[2] J.M. Maldacena, The Large $N$ limit of superconformal field theories and supergravity, Adv. Theor. Math. Phys. 2 (1998) 231 [hep-th/9711200] [INSPIRE]. 
[3] E. Witten, Anti-de Sitter space and holography, Adv. Theor. Math. Phys. 2 (1998) 253 [hep-th/9802150] [INSPIRE].

[4] S.S. Gubser, I.R. Klebanov and A.M. Polyakov, Gauge theory correlators from noncritical string theory, Phys. Lett. B 428 (1998) 105 [hep-th/9802109] [INSPIRE].

[5] J. Zaanen, Y.-W. Sun, Y. Liu and K. Schalm, Holographic Duality in Condensed Matter Physics, Cambridge University Press, Cambridge U.K. (2015).

[6] S.A. Hartnoll, A. Lucas and S. Sachdev, Holographic quantum matter, arXiv:1612.07324 [INSPIRE].

[7] J. Zaanen, Lectures on quantum supreme matter, arXiv:2110.00961 [INSPIRE].

[8] E. Oh, Y. Seo, T. Yuk and S.-J. Sin, Ginzberg-Landau-Wilson theory for Flat band, Fermi-arc and surface states of strongly correlated systems, JHEP 01 (2021) 053 [arXiv: 2007.12188] [INSPIRE].

[9] J.N. Laia and D. Tong, A Holographic Flat Band, JHEP 11 (2011) 125 [arXiv:1108.1381] [INSPIRE].

[10] Y. Liu and Y.-W. Sun, Topological nodal line semimetals in holography, JHEP 12 (2018) 072 [arXiv: 1801.09357] [INSPIRE].

[11] Y. Seo, G. Song, Y.-H. Qi and S.-J. Sin, Mott transition with Holographic Spectral function, JHEP 08 (2018) 077 [arXiv:1803.01864] [INSPIRE].

[12] S. Chakrabarti, D. Maity and W. Wahlang, Probing the Holographic Fermi Arc with scalar field: Numerical and analytical study, JHEP 07 (2019) 037 [arXiv:1902.08826] [INSPIRE].

[13] T. Faulkner, N. Iqbal, H. Liu, J. McGreevy and D. Vegh, Charge transport by holographic Fermi surfaces, Phys. Rev. D 88 (2013) 045016 [arXiv: 1306.6396] [INSPIRE].

[14] H. Liu, J. McGreevy and D. Vegh, Non-Fermi liquids from holography, Phys. Rev. D 83 (2011) 065029 [arXiv:0903.2477] [INSPIRE].

[15] R. Jackiw and C. Rebbi, Solitons with Fermion Number 1/2, Phys. Rev. D 13 (1976) 3398 [INSPIRE].

[16] M.Z. Hasan and C.L. Kane, Topological Insulators, Rev. Mod. Phys. 82 (2010) 3045 [arXiv: 1002.3895] [INSPIRE].

[17] X.L. Qi and S.C. Zhang, Topological insulators and superconductors, Rev. Mod. Phys. 83 (2011) 1057 [arXiv: 1008. 2026] [INSPIRE].

[18] N. Iqbal and H. Liu, Real-time response in AdS/CFT with application to spinors, Fortsch. Phys. 57 (2009) 367 [arXiv:0903.2596] [InSPIRE].

[19] T. Faulkner, H. Liu, J. McGreevy and D. Vegh, Emergent quantum criticality, Fermi surfaces, and $A d S_{2}$, Phys. Rev. D 83 (2011) 125002 [arXiv:0907.2694] [InSPIRE].

[20] T. Faulkner, N. Iqbal, H. Liu, J. McGreevy and D. Vegh, From Black Holes to Strange Metals, arXiv: 1003.1728 [INSPIRE].

[21] M. Blake and D. Tong, Universal Resistivity from Holographic Massive Gravity, Phys. Rev. D 88 (2013) 106004 [arXiv:1308.4970] [INSPIRE].

[22] R.A. Davison, K. Schalm and J. Zaanen, Holographic duality and the resistivity of strange metals, Phys. Rev. B 89 (2014) 245116 [arXiv:1311.2451] [INSPIRE]. 
[23] M. Blake and A. Donos, Quantum Critical Transport and the Hall Angle, Phys. Rev. Lett. 114 (2015) 021601 [arXiv: 1406.1659] [INSPIRE].

[24] X.-H. Ge, Y. Tian, S.-Y. Wu, S.-F. Wu and S.-F. Wu, Linear and quadratic in temperature resistivity from holography, JHEP 11 (2016) 128 [arXiv:1606.07905] [INSPIRE].

[25] Y. Ahn, H.-S. Jeong, D. Ahn and K.-Y. Kim, Linear-T resistivity from low to high temperature: axion-dilaton theories, JHEP 04 (2020) 153 [arXiv: 1907.12168] [INSPIRE].

[26] B.-J. Yang, E.-G. Moon, H. Isobe and N. Nagaosa, Quantum criticality of topological phase transitions in three-dimensional interacting electronic systems, Nat. Phys. 10 (2014) 774 [arXiv: 1406.2766].

[27] G. Song, J. Rong and S.-J. Sin, Stability of topology in interacting Weyl semi-metal and topological dipole in holography, JHEP 10 (2019) 109 [arXiv: 1904.09349] [INSPIRE].

[28] J. Custers et al., The break-up of heavy electrons at a quantum critical point, Nature $\mathbf{4 2 4}$ (2003) 524

[29] S.-S. Lee, Low energy effective theory of Fermi surface coupled with U(1) gauge field in $2+1$ dimensions, Phys. Rev. B 80 (2009) 165102 [arXiv:0905.4532] [INSPIRE].

[30] S. Sur and S.-S. Lee, Chiral non-Fermi liquids, Phys. Rev. B 90 (2014) 045121 [arXiv: 1310.7543] [INSPIRE].

[31] M.A. Metlitski and S. Sachdev, Quantum phase transitions of metals in two spatial dimensions: I. Ising-nematic order, Phys. Rev. B 82 (2010) 075127 [arXiv:1001.1153] [INSPIRE].

[32] M.A. Metlitski and S. Sachdev, Quantum phase transitions of metals in two spatial dimensions: II. Spin density wave order, Phys. Rev. B 82 (2010) 075128 [arXiv: 1005.1288] [INSPIRE].

[33] I. Mandal and S.-S. Lee, Ultraviolet/infrared mixing in non-Fermi liquids, Phys. Rev. B 92 (2015) 035141 [arXiv:1407.0033] [INSPIRE].

[34] S.-S. Lee, Recent Developments in Non-Fermi Liquid Theory, Ann. Rev. Condensed Matter Phys. 9 (2018) 227 [arXiv:1703.08172] [INSPIRE].

[35] A.L. Fitzpatrick, S. Kachru, J. Kaplan and S. Raghu, Non-Fermi-liquid behavior of large- $N_{B}$ quantum critical metals, Phys. Rev. B 89 (2014) 165114 [arXiv:1312.3321] [InSPIRE].

[36] D.F. Mross, J. McGreevy, H. Liu and T. Senthil, A controlled expansion for certain non-Fermi liquid metals, Phys. Rev. B 82 (2010) 045121 [arXiv: 1003.0894] [INSPIRE].

[37] D. Harlow and H. Ooguri, Constraints on Symmetries from Holography, Phys. Rev. Lett. 122 (2019) 191601 [arXiv:1810.05337] [INSPIRE]. 